\title{
Edge dislocations as sinks for sub-nanometric radiation induced defects in $\alpha$-iron
}

\author{
N. Anento ${ }^{1}$, L. Malerba ${ }^{2}$ and A. Serra ${ }^{1}$ \\ ${ }^{1}$ Department of Civil and Environmental Engineering, Universitat Politècnica de Catalunya, Jordi \\ Girona 1-3, 08034 Barcelona, Spain \\ 2 SCK-CEN, Nuclear Materials Science Institute, Boeretang 200, B-2400, Mol, Belgium
}

\begin{abstract}
The role of edge dislocations as sinks for small radiation induced defects in bcc-Fe is investigated by means of atomistic computer simulation. In this work we investigate by Molecular Statics $(\mathrm{T}=\mathrm{OK})$ the interaction between an immobile dislocation line and defect clusters of small sizes invisible experimentally. The study highlights in particular the anisotropy of the interaction and distinguishes between absorbed and trapped defects. When the considered defect intersects the dislocation glide plane and the distance from the dislocation line to the defect is on the range between $2 \mathrm{~nm}-4 \mathrm{~nm}$, either total or partial absorption of the cluster takes place, leading to the formation of jogs. Residual defects produced during partial absorption pin the dislocation. By the calculation of stress-strain curves we have assessed the strength of those residues as obstacles for the motion of the dislocation, which is reflected on the unpinning stresses and the binding energies obtained. When the defect is outside this range, but on planes close to the dislocation glide plane, instead of absorption we have observed a capture process. Finally, with a view to introducing explicitly in kinetic Monte Carlo models a sink with the shape of a dislocation line, we have summarized our findings on a table presenting the most relevant parameters, which define the interaction of the dislocation with the defects considered.
\end{abstract}

Key words: point defects, edge dislocation, defect interaction, radiation damage, atomic simulation.

\section{Introduction}

Prolonged irradiation progressively introduces defects in crystalline materials such as metals, thereby producing evolving microstructural changes that impact the properties of the materials at all levels, including modifications of their mechanical response to loads. The construction of physics-based models capable of describing quantitatively this microstructural evolution, up to times or irradiation doses comparable with experiments and of relevance for technological 
applications, is, therefore, an important field of research that triggered a large amount of effort, especially in connection with iron alloys, which are the basic model materials for steels [1-7]. In these models, irrespective of the method applied, the story of the defects introduced by irradiation is followed from their generation to their disappearance. Defects are created in the form of vacancies and self-interstitials, or small clusters thereof, but they evolve to become voids or dislocation loops via diffusional processes. They do so in interplay with each other and with the pre-existing microstructure, mainly defined by dislocations and grain boundaries, as well as influenced by the alloying elements present in the matrix, that modify the mobility and the stability of the defects produced, especially their clusters.

In order for models of microstructure evolution under irradiation to be reliable, the migration, aggregation and dissociation of clusters of defects need to be described according to the correct physical mechanisms, using interaction and energy parameters that should be quantitatively sensible, because the kinetics of evolution predicted by the model will heavily depend on their values [1-7]. Likewise, it is essential that the absorption of defects by sinks should be correctly described in quantitative terms, because a correct prediction of the number of surviving defects depends crucially on whether the model reproduces the correct balance between defect creation and destruction.

What is mainly important to know about a sink of irradiation defects is: (i) within which volume close to the sink one can consider a mobile defect to be interacting with it and then to be absorbed; (ii) whether the absorbed defect loses completely its identity inside the sink (actual absorption) or remains identifiable, even though not able any more to escape from it (trapping); (iii) whether or not there is a possibility that the defect is de-trapped or re-emitted by the sink and, if so, with which frequency this may happen; (iv) whether the sink can continue to absorb/trap indefinitely or not.

Models of microstructure evolution such as those based on the object kinetic Monte Carlo approach [1, 3-7] require data on absorption by sinks not only for single-point defects or for large glissile loops, but in fact for mobile defects of all sizes. Self-interstitial clusters are a relatively fast migrating type of defect for any size, even though with size-dependent mechanisms [8 - 10]. Also small vacancy clusters are mobile defects that can approach dislocation lines by diffusion: trivacancies are, for example, as mobile as small self-interstitial clusters [10]. Even clusters of up to $\sim 10$ vacancies remain, in fact, non-negligibly mobile at temperatures of technological interest [11, 12]. Thus all these defects can reach sinks and interact with them during an irradiation process.

Rate theory models use, to describe the absorption of defects by sinks in a mean field approximation, theoretical expressions obtained from the solution of the relevant diffusion equation with appropriate boundary conditions [13, 14]. In most object kinetic Monte Carlo models of microstructural evolution under irradiation, these theoretical expressions are used to 
mimic the sink effect of dislocations through simpler sinks, typically spherical absorbers, the volume fraction of which is calibrated to correspond to a certain dislocation density, by equalling the corresponding theoretical expressions. It is known that these expressions are consistent with the object kinetic Monte Carlo simulations, and vice versa $[15,16]$. With a view to introducing explicitly a sink with the shape of a dislocation line, however, it becomes important to have more precise data about how defect clusters of any size interact with immobile dislocation lines. This will allow their absorption or trapping by this type of sink to be treated as correctly as possible, especially in terms of capture distance and interaction energy involved. In this work we therefore investigate for the first time, with atomistic techniques, the interaction between an immobile dislocation line and defect clusters of very small sizes that have not yet been considered, focusing on the case of the edge dislocation as sink. Specifically, a static mapping of interaction energy and relevant reactions has been produced for vacancy clusters (2, 3 and 6 vacancies), as well as for interstitial clusters (2, 3, 7 and 19 self-interstitial atoms). This mapping allows the quantitative identification of regions around the dislocation line where different types of interactions occur, which can in principle be exported to object kinetic Monte Carlo models. The range of the interactions and the atomic scale reactions between the dislocation and the considered defects do not allow the elasticity approach to be used, while atomistic computer simulations are suitable. The study highlights, in particular, the anisotropy of the interaction and provides a criterion to distinguish between absorbed and trapped dislocation loops.

\section{Simulation method}

The interaction between a $1 / 2<111>\{110\}$ edge dislocation and several submicroscopic defects in bcc-Fe has been studied by using Molecular Statics (MS) modelling techniques; with an Embedded Atom Method (EAM) interatomic potential for bcc-Fe developed by Mendelev et al. [17]. This potential has been fitted to ab initio data and has proven to be able to reproduce accurately the most relevant properties of irradiation-induced defects [18].

The potential energy of the system has been minimized by a combination of the conjugate gradient method and the quasi-dynamical relaxation, which damps the kinetic energy when it reaches an allowed maximum. A $1 / 2[111](1 \overline{1} 0)$ edge dislocation is constructed in the crystal with principal crystallographic axes $x, y, z$ oriented along [111],[ $\overline{1} \overline{1} 2]$ and [1 $\overline{1} 0]$ directions, respectively, with the Burgers vector $\mathbf{b}=1 / 2$ [111] parallel to the $x$ axis, using a model developed by Osetsky and Bacon [19] with periodicity along both the dislocation line and Burgers vector. The boundaries in the $z$ direction are made of rigid blocks that can move on the $x$ and $y$ directions to apply strains to the system. In this way, periodic boundary conditions are applied along the $x$ and $y$ directions. The defects studied are point defects, namely vacancy (V) and self-interstitial atom (SIA), and their clusters $\left(V_{n}, n \leq 6\right.$ and $m$ SIAs, $\left.m \leq 19\right)$, which have a diameter below $1 \mathrm{~nm}$. The 
interaction energy between two objects (A and B) is calculated according to the following expression:

$$
E_{I}(A, B)=\left[E_{T}(A B)+E_{T}(\text { perfect })\right]-\left[E_{T}(A)+E_{T}(B)\right]
$$

The values needed to calculate $E_{I}(A, B)$ are the total energies of the relaxed crystal containing either the defect $\mathrm{A}, \mathrm{B}$ or the complex $\mathrm{AB}$, where $E_{T}$ (perfect) is the total energy of a perfect crystal. According to this definition for $E_{I}(A, B)$, a negative value of the interaction energy implies an attractive interaction between defects $\mathrm{A}$ and $\mathrm{B}$. The binding energy is defined as $-E_{I}(A, B)$. Using the above equation, we have studied cases where the point-defect cluster is located in the position with the maximum binding energy to the dislocation.

We have considered the lowest energy configurations of the point-defect clusters of interest [20, 21]. The stable configurations of vacancy clusters in bcc-Fe are taken from figure 9a in ref. [21]. For the interstitial clusters, the stable $<110>$ configurations of the 2SIA and 3SIA clusters are those presented in [9]. The 7SIA and 19SIA clusters are formed by $<111>$ crowdions with hexagonal shape and Burgers vector $1 / 2<111>$. For these defects there are two possible interaction scenarios, depending on the orientation of their Burgers vectors, namely, parallel or inclined with respect to the Burgers vector of the dislocation. Four different simulation boxes have been used, denoted as $A-D$, the dimensions of which are shown in Table 1 and are sufficient to avoid the interaction of the clusters with their replicas during relaxation. To this end, the length along the $x$ direction has been increased in crystal C (parallel geometry) and D (non-parallel geometry). The smallest crystal, named A, has been used for the $V_{n}$ and $n$ SIA clusters with $n \leq 3$, while crystal B was used for the simulation of the $V_{6}$ cluster.

\begin{tabular}{|c|c|c|c|}
\hline & $\mathrm{L}_{x}(\mathrm{~nm})$ & $\mathrm{L}_{\mathrm{y}}(\mathrm{nm})$ & $\mathrm{L}_{z}(\mathrm{~nm})$ \\
\hline Crystal A & 37.2 & 3.5 & 20.6 \\
\hline Crystal B & 37.2 & 7.7 & 20.6 \\
\hline Crystal C & 74.3 & 7.7 & 20.6 \\
\hline Crystal D & 111.4 & 7.7 & 20.6 \\
\hline
\end{tabular}

Table 1. Dimensions in $\mathrm{nm}$ of the crystals used in this work

To distinguish between absorbed and trapped defects and to investigate the effect of $\mathrm{V}_{6}, 7 \mathrm{SIA}$ and 19SIA clusters on the mobility of the edge dislocation, incremental strains followed by relaxation have been subsequently applied to the system, up to the complete release of the dislocation. The stress-strain curves were obtained from crystals with dislocation lines of $7.7 \mathrm{~nm}$, which is, in turn, the distance between a cluster and its images due to periodic boundary conditions. 


\section{Results}

In general, as predicted by the elasticity theory, the attractive interaction and possible reaction between the dislocation and clusters occurs in the compression region of the edge dislocation for vacancy clusters and in the tension region of the edge dislocation for SIA clusters. However, in the core of the dislocation, where the elasticity theory fails, the precise reaction should be described by atomistic simulation.

In the present work the simulation has been done at $0 \mathrm{~K}$, thus only athermal spontaneous recombination is produced. Under these conditions, only the straight dislocation and <111> type clusters are able to glide during the relaxation process, if they are within the interaction distance. Clusters formed by vacancies or by 2 - 3 SIAs do not move towards the dislocation during the interaction process, but can experience local displacements, when they are in the vicinity of the dislocation core.

The energies released when a point defect is absorbed by the dislocation calculated with the same EAM potential are $0.85 \mathrm{eV}$ for a vacancy and $2.85 \mathrm{eV}$ for an SIA. These are the reference values considered for the calculation of the binding energy of the trapped or partially absorbed defects, as described below.

\subsection{Vacancy clusters $\left(V_{2}, V_{3}\right.$ and $\left.V_{6}\right)$}

Figure 1a presents a scheme of the planes parallel to the dislocation glide plane (DGP hereafter); the colours of the planes will be used in the curves of figures 2 and 4 to indicate the location of the reference point defect for each cluster. Figure $1 \mathrm{~b}$ presents the configurations of vacancy clusters indicating the reference vacancy. These configurations are the most stable for both DFT calculations and the present EAM potential $[20,21]$. For a given configuration there are

of course several possible orientations relative to the dislocation line. Here we present the results for the orientations more representative of the interaction energy and capture distance, with a view to providing parameters for object kinetic Monte Carlo models.

In the lowest energy configuration for the $\mathrm{V}_{2}$ cluster, the two vacancies are at $2^{\text {nd }}$ nearestneighbour distance along a [100] direction. In our calculations, the $V_{2}$ has been located parallel to the DGP; $a V_{3}$ cluster is formed from $V_{2}$, adding a vacancy, in the first nearest neighbour shell of both others, on the plane above or below. Finally, $V_{6}$ is an octahedron formed by 4 vacancies in a plane parallel to the DGP and two vacancies located in opposite sites, perpendicular to the DGP. 


\subsubsection{Interaction energy and capture distance}

Figure 2 presents the interaction energy of the $\mathrm{V}_{2}$ cluster with the edge dislocation as a function of the distance along the Burgers vector direction and the location of the cluster in relation to the DGP. Negative values represent attraction and/or absorption.

Preliminary calculations with the single vacancy had shown that this defect, when interacting with an edge dislocation, is annihilated athermally only when it is located on the DGP. Similarly, the cluster $V_{2}$ is completely absorbed by the dislocation only when located at the glide plane (red curve in Figure 2). For larger clusters this is not necessarily the case and the recombination is not always associated with the vacancies at the DGP. Notice that the red curve in Figure 2 has two symmetric discontinuities and the points between these correspond roughly to the same energy. All these points represent the same final relaxed configuration because, when the cluster is located at the capture distance, the dislocation moves up to the $\mathrm{V}_{2}$ cluster and absorbs it. After absorption the dislocation line has a double jog. The maximum binding energy for a $V_{2}$ cluster that is not absorbed and maintains its individuality is $0.7 \mathrm{eV}$ and occurs at the plane above the glide plane, i.e., at the compression side. This is indicated in Table 2, where the relative position of vacancy clusters, the type of interaction and the corresponding binding energy are summarized. There is a slight attraction of the $V_{2}$ cluster when located at the first plane in the tension region as shown in Figure 2 (cyan points). In this case the cluster is trapped by the dislocation but it is released when the dislocation moves under an applied shear stress. In accordance with the small binding energy, the stress necessary to release the dislocation from the $\mathrm{V}_{2}$ cluster is only $96 \mathrm{MPa}$, i.e. only $10 \mathrm{MPa}$ higher than the Peierls stress, i.e., $85 \mathrm{MPa}$.

The $V_{3}$ cluster intersects the DGP either with one vacancy (with the other two lying in the compression region) or two vacancies (with the third one lying in the tensile region). In the latter case both vacancies are annihilated and the remaining vacancy has a binding energy of $0.4 \mathrm{eV}$. In the former, there is no recombination, obtaining a binding energy of $1.5 \mathrm{eV}$ for the whole cluster, implying that this cluster can be a relatively strong obstacle for the motion of the dislocation. When a $\mathrm{V}_{6}$ cluster intersects the DGP by the upper vertex (one vacancy), the other five vacancies are in the tensile region. In this case there is no recombination and the binding energy is about $0.9 \mathrm{eV}$. The maximum binding is obtained when the cluster intersects de DGP by the plane containing 4 vacancies. Two of the vacancies are absorbed leaving a double jog on the dislocation line and $\mathrm{a} \mathrm{V}_{4}$ cluster is trapped with a binding energy of $1.2 \mathrm{eV}$. The capture distance for all clusters in the vicinity of the glide plane is $5-6 a_{0}$ ( $a_{0}$ is the lattice parameter), i.e., 1.5 to $1.7 \mathrm{~nm}$, along the Burgers vector direction. Within this distance the dislocation moves towards the cluster during the relaxation process.

There is no annihilation of vacancies at the compression region of the dislocation when any vacancy of the cluster is located away from the DGP. The interaction region (binding energy > 
$0.1 \mathrm{eV})$, is extended up to $4 \mathrm{a}_{0}(1.15 \mathrm{~nm})$ in the Burgers vector direction, as shown schematically in Figure $3 a$, and about 1.7, 1.9 and $3.2 \mathrm{~nm}$ above the glide plane for $V_{2}, V_{3}$ and $V_{6}$ respectively. The interaction in the tensile region of the dislocation is negligible.

\subsubsection{Unpinning stress}

To elucidate whether the clusters are trapped but retain their nature upon the interaction with the dislocation, or they are absorbed, the system has been subjected to an incremental strain followed by relaxation, until the complete release of the dislocation.

The stress-strain curves are calculated for a dislocation length of $7.7 \mathrm{~nm}$. They show an elastic deformation followed by a drop of stress when the dislocation is attracted by the cluster and a further increase of stress if the dislocation is pinned by the remaining vacancies; once the remaining defect is detached, the stress drops up to a value close to the Peierls stress of the edge dislocation (85 MPa). Figure $3 \mathrm{~b}$, corresponding to $\mathrm{V}_{6}$, is presented as an example. It is important to note that the unpinning stress, presented in Table 2 , is almost proportional to the binding energy of the remaining defect trapped by the dislocation, as shown in Figure 3c. In the case (II), the 4 vacancies that are not spontaneously absorbed form a $V_{4}$ cluster that is left behind the dislocation after detachment.

Note that the high value of the unpinning stress is due to the high line density of obstacles that interact with the dislocation through the periodic boundary conditions. Since the reaction of the cluster with the dislocation is produced prior to the application of a shear stress, increasing the length of dislocation line between clusters would diminish the unpinning stress but would leave the reaction of the cluster unchanged.

\begin{tabular}{|c|c|c|c|c|c|}
\hline Cluster & $\begin{array}{l}\text { Cluster position } \\
\text { relative to disl. }\end{array}$ & Interaction & $E_{b}(e V)$ & $\Delta$ Eabs (eV) & $\begin{array}{l}\text { Unpinning } \\
\text { Stress (MPa) }\end{array}$ \\
\hline$V_{1}$ & - & Absorption & - & - & 90 \\
\hline$V_{2}$ & $-O$ & Absorption & - & - & 104 \\
\hline$V_{2}$ & iii) & Trapped & 0.7 & $0.85 \times 2=1.7$ & 195 \\
\hline$V_{3}$ & i) & $\begin{array}{l}2 \mathrm{~V} \text { absorbed } \\
\text { \&weak trap }\end{array}$ & 0.4 & 0.85 & 161 \\
\hline$V_{3}$ & ii) & Trapped & 1.5 & $0.85 \times 3=2.55$ & 308 \\
\hline$V_{6}$ & i) & Trapped & 0.9 & $0.85 \times 6=5.1$ & 217 \\
\hline$V_{6}$ & ii) & $\begin{array}{c}2 \text { V absorbed } \\
\text { \&trap }\end{array}$ & 1.2 & $0.85 \times 4=3.4$ & 281 \\
\hline$V_{6}$ & iii) & $\begin{array}{c}5 \mathrm{~V} \text { absorbed } \\
\text { \&trap }\end{array}$ & 0.8 & 0.85 & 189 \\
\hline
\end{tabular}


Table 2. Interaction <dislocation- $V_{n}>$. The location of the dislocation is indicated by the black line. The compression region of the dislocation is above the line. The Burgers vector of the dislocation is perpendicular to the paper.

The second last column in Table 2 gives the energy gain by absorbing the remaining vacancies in the dislocation. Clearly, complete absorption corresponds to the lowest energy state in all cases, with the only questionable case of the single vacancy that survives the absorption of 5 out of 6 vacancies in $\mathrm{V}_{6}$. This suggests that, even if there is an energy barrier preventing complete athermal absorption, eventually also the surviving vacancies will be absorbed at finite temperature. The energy for the surviving vacancies to migrate away from an immobile dislocation would in all cases be between 1 and $2 \mathrm{eV}$, so detrapping can be excluded (see [11] for $V$ cluster migration energy values, to be summed to the binding to obtain the dissociation energy). Thus, residual defects initially trapped at the dislocation could survive only if the dislocation is immediately after unpinned by an external stress.

\subsection{Self-interstitial atom clusters $(2,3,7$ and 19 interstitials)}

The most stable clusters up to 5 SIAs are formed by parallel $<110>$ dumbbells that move three dimensionally or in a mixed one-three-dimensional fashion. Mobile clusters of 6 SIAs or more, in contrast, stabilise as parallel $<111>$ crowdions that perform one-dimensional motion along the crowdion line $[8,9]$. In this paper we discuss the interaction of clusters of 2 and 3 SIAs as representative of the former class and clusters of 7 and 19 SIAs as representative of the latter.

- Clusters formed by $<110>$ dumbbells.

A 2SIA cluster has been constructed with a couple of $\langle 110\rangle$ dumbbells oriented parallel to the DGP and separated by a $1^{\text {st }}$ nearest neighbour distance. The most stable configuration of a 3SIA cluster is obtained by adding an extra $<110>$ dumbbell to a 2 SIA cluster, giving to the defect a triangular shape (see Table 7 in [18]).

The interaction energy of a 2SIA cluster with the edge dislocation is plotted in Figure 4a, where the points representing athermal absorption are encircled. Thus, absorption is produced when the cluster is at the first plane below the DGP (pale blue curve), at the DGP (red curve) and at the first plane above the DGP. The last of these three positions results in a repulsive interaction of $0.4 \mathrm{eV}$ at a distance $\mathrm{a}_{0}$ from the dislocation core, but for shorter distances the relaxation process leads to a reorientation of the cluster towards the tension region, finally resulting in the cluster being absorbed. This reorientation is favoured by the high mobility of the interstitial and its translation-rotation mechanism of motion. The other positions at the tensile side results in a positive binding without athermal absorption, with a maximum value of $1.8 \mathrm{eV}$ (Figure $4 \mathrm{~b}$ ). $3 \mathrm{SIA}$ clusters are absorbed when located at the first plane below the DGP (tension region) and the maximum binding energy for clusters not absorbed is $3.2 \mathrm{eV}$ (Figure $4 \mathrm{~b}$ ). The absorption distance 
along the dislocation Burgers vector is $2 a_{0}$ for both clusters. At the compression region there is a repulsive interaction energy. In this case, too, the energy gain when the dislocation absorbs the interstitials is larger than the binding energy, so one should conclude that eventually they will be absorbed.

\section{- $\quad$ Clusters formed by $<111>$ crowdions.}

The interaction with a dislocation for clusters with radius above $1 \mathrm{~nm}$ has been reported in the literature, see for example [22 - 25]. For a cluster with a radius of $0.7 \mathrm{~nm}$, Osetsky et al [23] have shown that the isotropic elasticity theory seems to describe the long-range interactions quite well, even with the infinitesimal loop approximation, but fails to describe the short-range interactions, where the atomic structure should be considered explicitly. In the following we describe the interaction of two of the smallest clusters formed by $<111>$ crowdions with the edge dislocation.

Figure 5a shows a scheme of the simulated system. The 1-D motion of these clusters determines the interactions with the edge dislocation. If the Burgers vector of the cluster is inclined relative to the DGP, the absorption of the cluster occurs when the cluster is at a distance of the order of $2 \mathrm{~nm}$ and $4 \mathrm{~nm}$ for clusters of 7 and 19SIA respectively. The reaction implies a reorientation of the Burgers vector of the cluster and the final result is a dislocation with a double jog, as shown in Figure $5 \mathrm{~b}$. Out of this region of spontaneous athermal recombination there is a maximum binding energy of 1.2 and $3.1 \mathrm{eV}$ for the 7 and 19SIAs respectively.

Clusters with Burgers vector parallel to the one of the dislocation, in contrast, move perpendicular to the dislocation line and keep their distance, $\mathrm{H}$, constant, from the cluster centre to the glide plane. Total or partial absorption occurs when the cluster is in the tension region of the dislocation at a distance $H$ ranging from zero to $R+\sqrt{3} a_{0}$, where $R$ is the cluster radius. The absorption distance along the Burgers vector direction is thus $2.7 \mathrm{~nm}$ and $4 \mathrm{~nm}$ for the 7 and 19SIA respectively. Clusters below this region are attracted by the dislocation, without being absorbed, with a maximum binding energy of $4.5 \mathrm{eV}(7 \mathrm{SIA})$ and 9.7eV (19SIA), as shown in Figure 6 where the binding energy is plotted versus the distance $\mathrm{H}$ along the direction perpendicular to the DGP. For distances $\mathrm{H}$ much bigger than the loop radius, i.e., $\mathrm{H}>5 \mathrm{~nm}$, the energy compares well with the theoretical estimation of the loop-dislocation interaction energy calculated in an isotropic elastic medium obtained in [26]. The same comparison was reported in [23] for a 37SIA cluster. For shorter distances the elastic approach underestimates the interaction energy.

Clusters at the compression region are repelled by the dislocation. Similar reactions can be found in hcp zirconium [27]. 
The partial absorption of the cluster produces residual defects that pin the dislocation. As with vacancy clusters, in order to distinguish between absorption and trapping, the system has been subjected to an external stress up to dislocation unpinning. Table 3 presents the positions of clusters prior to the absorption, the residual defects after unpinning and the unpinning stress from a $7.7 \mathrm{~nm}$ dislocation line.

We describe here only the reactions for the 7SIAs cluster, because the reactions for the 19SIAs are equivalent. When $\mathrm{H}=0.6 \mathrm{~nm}$ there is only one (110) type plane between the cluster and the dislocation line. For the cluster to be fully absorbed, one more interstitial is needed to fill the gap between the cluster and the dislocation line. This is obtained from a Frenkel pair created during the application of the external stress. As a result, a vacancy is left behind. $\mathrm{H}=0.4 \mathrm{~nm}$ corresponds to the cluster tangent to the dislocation line therefore the absorption is the simplest. For $\mathrm{H}=(0.2$ or 0 ) $\mathrm{nm}$, the centre of the cluster is near (or at) the glide plane and 5 (or 2) out of 7 SIAs are absorbed. The remaining ( 2 or 5 ) SIAs are in the compression region and are repelled by the dislocation.

\begin{tabular}{|c|l|l|l|}
\hline (nSIA) & $\begin{array}{l}\mathrm{H} \\
(\mathrm{nm})\end{array}$ & $\begin{array}{l}\text { Residual } \\
\text { defects }\end{array}$ & $\begin{array}{l}\text { Unpinning } \\
\text { stress (MPa) }\end{array}$ \\
\hline 7 & 0.6 & $\mathrm{~V}$ & 450 \\
\hline 7 & 0.4 & None & 118 \\
\hline 7 & 0.2 & 2SIA & 196 \\
\hline 7 & 0 & 5SIA & 107 \\
\hline 19 & 0.8 & $\mathrm{~V}_{2}$ & 310 \\
\hline 19 & 0.6 & $2 \mathrm{~V}$ & 322 \\
\hline 19 & 0.4 & None & 125 \\
\hline 19 & 0.2 & 7SIA & 107 \\
\hline 19 & 0 & 12SIA & $97 / 125$ \\
\hline
\end{tabular}

Table 3. Interaction (with total or partial absorption) between an edge dislocation and nSIA clusters $(n=7,19)$. $H$ is the distance from the dislocation to the centre of the cluster located at the tension region of the dislocation. The stress is the one needed for the reaction and unpinning of the dislocation.

\subsection{Summary of results and possible usage in kinetic Monte Carlo simulations}

In Table 4 the maximum binding energy of defects and ranges when they are not absorbed by the dislocation are presented, and compared with the corresponding energy release if an equal 
number of single point-defects is absorbed by the dislocation. Vacancies interact mainly at the compression region and clusters of interstitials interact mainly at the tension region of the dislocation. For the clusters of 7 and 19 SIAs the relative orientation, i.e. parallel or inclined with respect to the dislocation line, is indicated. These results are complemented by the results in tables 2 and 3, where the trapping and absorption of defects is indicated.

\begin{tabular}{|c|c|c|c|}
\hline Defect & Binding energy (eV) & $\Delta$ Eabs (eV) & Range (nm) [111] / [1-10] \\
\hline $\mathrm{V}_{2}$ & 0.7 & 1.7 & $1.3 / 1.7$ (compression) \\
\hline $\mathrm{V}_{3}$ & 1.5 & 2.55 & $1.7 / 2.0$ (compression) \\
\hline $\mathrm{V}_{6}$ & 1.2 & 5.1 & $2.1 / 3.3$ (compression) \\
\hline 2SIA & 1.8 & 5.7 & 2.0 (tension) \\
\hline 3SIA & 3.2 & 8,55 & 2.0 (tension) \\
\hline 7SIA & $\begin{array}{c}4.5 \text { parallel /1.2 } \\
\text { Inclined }\end{array}$ & $\sim 20$ & $\begin{array}{c}(2.7 / 4.0) \text { parallel / }>2.5 \\
\text { Inclined }\end{array}$ \\
\hline 19SIA & $\begin{array}{c}9.7 \text { parallel/ 3.1 } \\
\text { Inclined }\end{array}$ & $\sim 54$ & Inclined \\
\hline
\end{tabular}

Table 4. Summary of the maximum binding energy cluster-dislocation for non-absorbed clusters and the range of interaction along the Burgers vector direction, [111], and the direction perpendicular to the DGP, [1-10]. The energy release upon absorption is also indicated.

The results of the present work suggest that the interaction of small mobile defect clusters with immobile dislocation lines is a complex process that is influenced by strictly atomistic details that the elasticity theory would not be able to catch. Simulating the absorption of these clusters at dislocation lines in non-atomistic models, such as object kinetic Monte Carlo [1-7], is therefore challenging. A first approach could be, of course, to describe the interaction between mobile defects and dislocation line in terms of elastic interaction, at least at sufficiently large distance. However, this approach will anyway oblige to introduce a number of approximations, because while for single point-defects and for voids or loops, as well as for dislocation lines, there are reasonable descriptions of the elastic field, this is not necessarily true for small point-defect clusters, that should be described simply as Eshelby inclusions. Importantly, moreover, the atomic level details of the interaction could not be grasped, thus anyway the final outcome of the reaction should be based on atomistic considerations. In addition, the long-range nature of the 
elastic interaction will increase the computational load and this, in some cases, may play as a strongly limiting factor for the application of a microstructure evolution model to simulate specific experiments. Thus, more simplified approaches that still have some foundation in atomistic results can be useful.

In the case of the edge dislocation that is studied here, it is possible to simplify the picture by distinguishing compression and tension sides, the former absorbing or trapping only vacancy clusters, the latter absorbing or trapping only interstitial clusters. It is accordingly possible to imagine that the cylinder that could simulate the dislocation line will have two halves, separated by the glide plane on which the Burgers vector lies: one interacting only with vacancy-type defects, the other only with interstitial-type defects. The vacancy half-cylinder could have a crosssection similar to the one schematically depicted in Figure 3a: defects that end up inside the halfcylinder volume will be absorbed, given that the energies involved suggest that finally all vacancies of a captured cluster will be absorbed. The absorption radius (or semi-axis in an elliptical cross-section) may increase moderately with the size of the cluster, or an effective average value may be considered. The definition of an external region where a binding $<0.1 \mathrm{eV}$ exists may also be defined. The interstitial half-cylinder, in contrast, is a more complex object. The interaction will depend on the size of the clusters and whether the mobile dislocation loop has its Burgers vector parallel to the glide plane or inclined with respect to it. Small SIA clusters (less than 5) and loops that come within a half-cylinder of a certain smaller radius are absorbed, while a halfcylinder with a larger radius will define a region where the loops (though not the smaller clusters) can be trapped, with a certain effective value of trapping energy. However, both the radius and the trapping energy effective value will depend on whether the Burgers vector of the loop is parallel to the one of the dislocation or not, as well as on the loop size (for small SIA clusters an effective range of $\sim 2 \mathrm{~nm}$ can be adopted). Of course the application of a recipe of this type, on which several variations are possible, will always correspond to a strong approximation. Yet this approximation will retain some physical information, while being very much in line with those that are most commonly used to define the parameters of microstructure evolution models, whenever these aim at a direct comparison with the results of microstructural characterisation of irradiated materials.

\section{Conclusions}

The interaction of small point defect clusters with an edge dislocation of Burgers vector $1 / 2<111>$ has been studied by molecular static simulation ( $T=0 \mathrm{~K})$. The results are intended to supply more accurate inputs to the object kinetic Monte Carlo simulations that performs the evolution of the microstructure under electron and neutron irradiation.

Under this perspective, the following conclusions are derived: 
i) Di-vacancies can be absorbed by the dislocation athermally but the other vacancy clusters are only partially absorbed at $\mathrm{T}=\mathrm{OK}$

ii) The binding energy between dislocation and vacancy clusters ranges from 0.7 to $1.5 \mathrm{eV}$. The interaction energy is significant only at the compression region of the dislocation and the range increases with the number of vacancies up to $3.3 \mathrm{~nm}$ in the direction perpendicular to the glide plane.

iii) The binding energy between the dislocation and interstitial clusters with 2 and 3 SIA depends on the cluster size and is much higher compared to vacancy clusters. The interaction occurs mainly in the tension region of the dislocation, but the higher mobility of the interstitial clusters favours the migration of clusters from the compression region to the tension region increasing the efficiency of the dislocation as sink for the smaller interstitial clusters. The range of interaction is about $2 \mathrm{~nm}$ in the tension region.

iv) Clusters bigger than 5SIAs have a one-dimensional motion. If their Burgers vector is not parallel to the one of the dislocation, they are absorbed by the dislocation within a distance of $2.5 \mathrm{~nm}(7 \mathrm{SIA})$ and $4.5 \mathrm{~nm}$ (19SIA). In contrast, only a small fraction of the clusters with parallel Burgers vector are absorbed. In general, they decorate the tension part of the dislocation with a maximum binding energy of 4.5eV (7SIA) and 9.7eV (19SIA).

v) Clusters with Burgers vector parallel to the one of dislocation can be absorbed totally or partially as a function of their position relative to the dislocation core. If clusters are partially absorbed they pin the dislocation. Under an external stress the dislocation can be unpinned and some remaining defects are released.

These conclusions allow simple but still physical schemes to simulate the absorption and trapping of defect clusters by dislocation lines acting as sinks.

\section{Disclaimer.}

This work reflects only the author's view and that the European Commission is not responsible for any use that may be made of the information it contains.

\section{Acknowledgments.}

This work has been partially supported by the Spanish MINECO (FIS2015-69017-P). This project has received funding from the Euratom research and training programme 2014-2018 under grant agreement No 661913 (SOTERIA). This work also contributes to the Joint Program on Nuclear Materials (JPNM) of the European Energy Research Alliance (EERA) 


\section{References:}

[1] R. E. Stoller, S. I. Golubov, C. Domain, C. S. Becquart, J. Nucl. Mater. 382 (2008) 77.

[2] E. Meslin, A. Barbu, L. Boulanger, B. Radiguet, P. Pareige, K. Arakawa, C. Fu, J. Nucl. Mater. $382(2008) 190$.

[3] C. S. Becquart, A. Barbu, J. Bocquet, M.J. Caturla, C. Domain,C.C. Fu, S. I. Golubov, M. Hou, L. Malerba, C. Ortiz, A. Souidi, R.E. Stoller, J. Nucl. Mater. 406 (2010) 39.

[4] N. Soneda, S. Ishino, A. Takahashi, K. Dohi, J. Nucl. Mater. 323 (2003) 169.

[5] V. Jansson, M. Chiapetto, L. Malerba, J. Nucl. Mater. 442 (1) (2013) 341.

[6] V. Jansson, L. Malerba, J. Nucl. Mater. 443 (1) (2013) 274.

[7] V. Jansson, L. Malerba, J. Nucl. Mater. 452 (1) (2014) 118.

[8] D.A. Terentyev, L. Malerba, M. Hou, Phys. Rev. B 75 (2007) 104108.

[9] N. Anento, A. Serra, Yu.N. Osetsky, Model. Simul. Mater. Sci. Eng. 18 (2010) 025008.

[10] C. C. Fu, F. Willaime, C.R. Physique 9 (2008) 335.

[11] M.I. Pascuet, N. Castin, C.S. Becquart, L. Malerba, J. Nucl. Mater. 412 (2011) 106.

[12] N. Castin, M.I. Pascuet, L. Malerba, J. Nucl. Mater. 429 (2012) 315.

[13] F. Nichols, J. Nucl. Mater. 75 (1978) 32.

[14] H. Trinkaus, H. L. Heinisch, A.V. Barashev, S.I. Golubov, B. N. Singh, Phys. Rev. B 66 (2002) 060105.

[15] L. Malerba, C. S. Becquart, C. Domain, J. Nucl. Mater. 360 (2007) 159.

[16] V. Jansson, L. Malerba, A. De Backer, C.S. Becquart, C. Domain, J. Nucl. Mater. 442 (2013) 218.

[17] M.I. Mendelev, S. Han, D.J. Srolovitz, G.J. Ackland, D.Y. Sun, and M. Asta, Philos. Mag. 83 (2003) 3977

[18] L. Malerba, et al., Journal of Nuclear Materials 406 (2010) 19].

[19] Yu.N. Osetsky and D.J. Bacon Model. Simul. Mater. Sci. Eng. 11 (2003) 427.

[20] C. C.Fu, J. Dalla Torre, F. Willaime, J.L. Bocquet and A. Barbu, Nature Materials, 4 (2005) 68 
[21] D. Kulikov, L. Malerba and M. Hou, Philos. Mag. 86 (2006) 141.

[22] N.M. Ghoniem, B.N Singh, L.Z. Sun, T. Díaz de la Rubia, J. Nucl. Mater. 276 (2000) 166.

[23] Yu.N. Osetsky, D.J. Bacon, F. Gao, A. Serra and B.N. Singh, J. Nucl. Mater. 283-287 (2000) 784.

[24] D.J.Bacon, Yu.N. Osetsky and Z.Rong, Philos. Mag. 86 (2006) 3921.

[25] D. Terentyev, L. Malerba, D.J. Bacon and Yu.N. Osetsky, J. Phys.: Condens. Matter 19 (2007) 456211.

[26] A.J.E. Foreman, AERE Report - R 4654, (1964)

[27] A. Serra, D.J. Bacon, Model. Simul. Mater. Sci. Eng. 21 (2013) 045007

\section{Figure captions}

Figure 1. a) Scheme of the planes parallel to the dislocation glide plane (red). b) Configuration of vacancy clusters. The position of the reference vacancy is the one plotted in figure 2

Figure 2. Interaction energy of $V_{2}$ with the edge dislocation versus the position of $V_{2}$ along the [111] direction. The colours of the curves refer to the position of the planes in figure 1a. Points encircled represent spontaneous absorption. Above and below refer to the dislocation glide plane, i.e., 'above' and 'below' correspond to compression and tension regions of the dislocation respectively.

Figure 3. a) Schematics of the interaction region (binding energy $>0.1 \mathrm{eV}$ ) for vacancy clusters up to 6 vacancies. b) Stress-strain curves of a system formed by the edge dislocation and a $V_{6}$ cluster. The three curves correspond to the positions indicated in table 2. c) Unpinning stress of defects versus the defect-dislocation binding energy.

Figure 4. Interaction energy of 2SIA cluster with the edge dislocation versus the cluster position along the [111] direction. The colours of the curves refer to the position of the planes in figure 1a. Points encircled represent spontaneous absorption. Above and below refer to the dislocation glide plane, as in the caption of figure 2.

Figure 5. a) Schematics of the interaction of an edge dislocation with clusters of 7 and 19SIA. b) Dislocation line after the absorption of the 19SIA cluster. The atoms represented are chosen by their coordination number.

Figure 6. Binding energy of clusters of 7 and 19 SIA with Burgers vector parallel to the dislocation glide plane as a function of the distance from the centre of the cluster to the dislocation line. 


\section{Schematic of the [111] lines investigated}

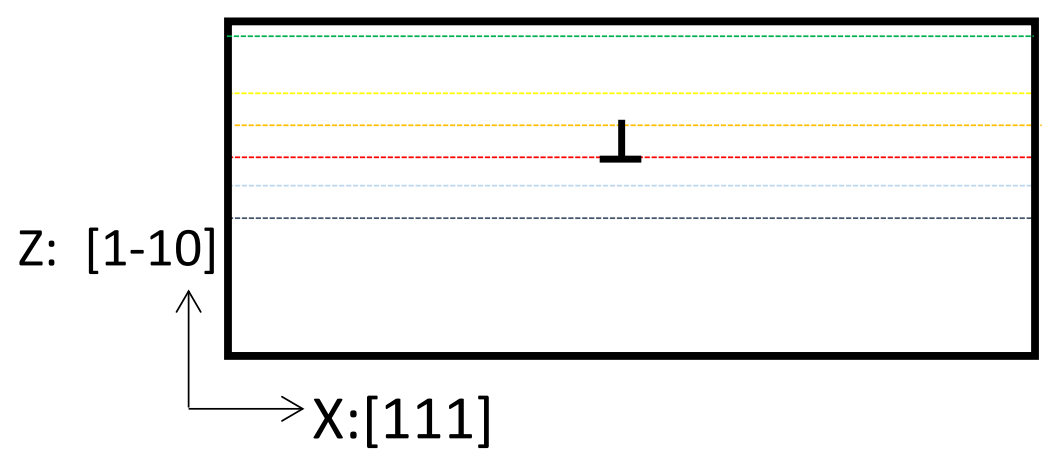

\section{Vacancy cluster configurations}

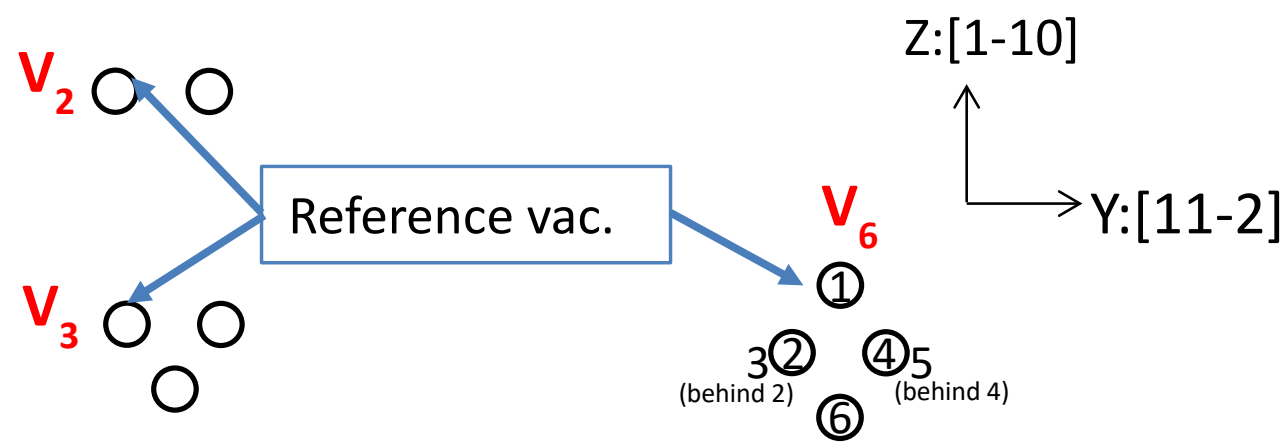

Figure 1. a) Scheme of the planes parallel to the dislocation glide plane (red). b) Configuration of vacancy clusters. The position of the reference vacancy is the one plotted in figure 2 


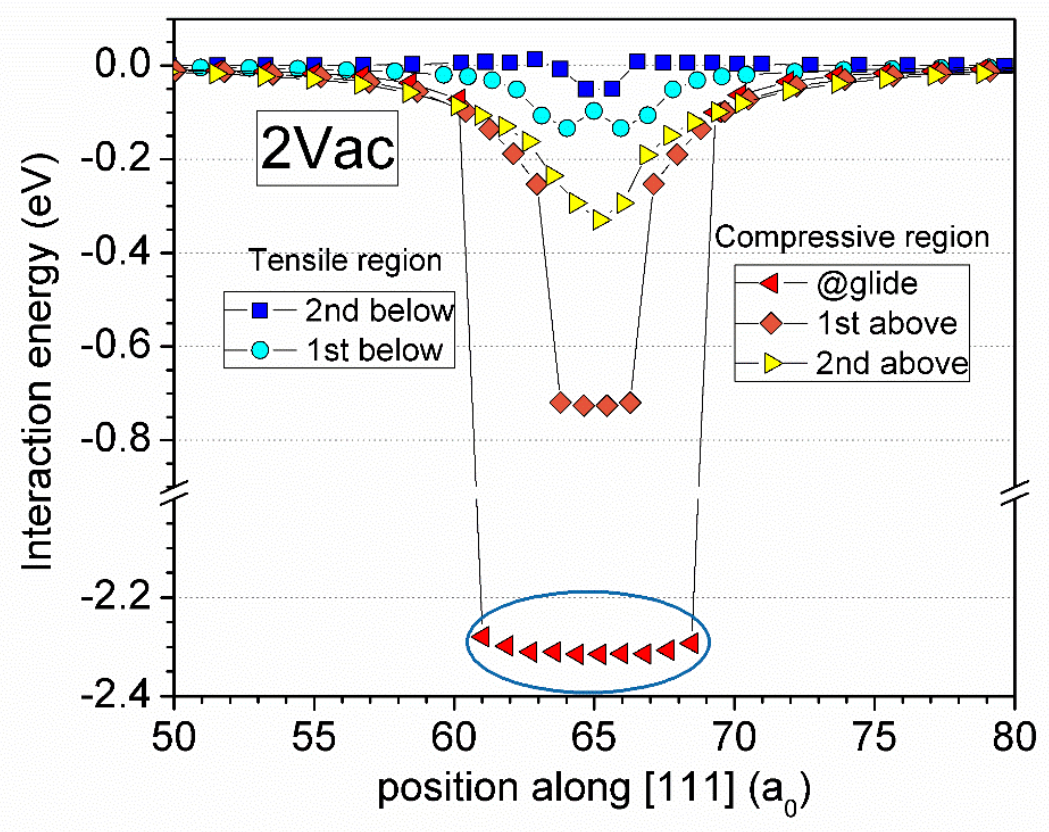

Figure 2. Interaction energy of $\mathrm{V}_{2}$ with the edge dislocation versus the position of $\mathrm{V}_{2}$ along the [111] direction. The colours of the curves refer to the position of the planes in figure 1a. Points encircled represent spontaneous absorption. Above and below refer to the dislocation glide plane, i.e., 'above' and 'below' correspond to compression and tension regions of the dislocation respectively. 


\section{$\mathrm{E}_{\mathrm{b}}<0.1 \mathrm{eV}$}
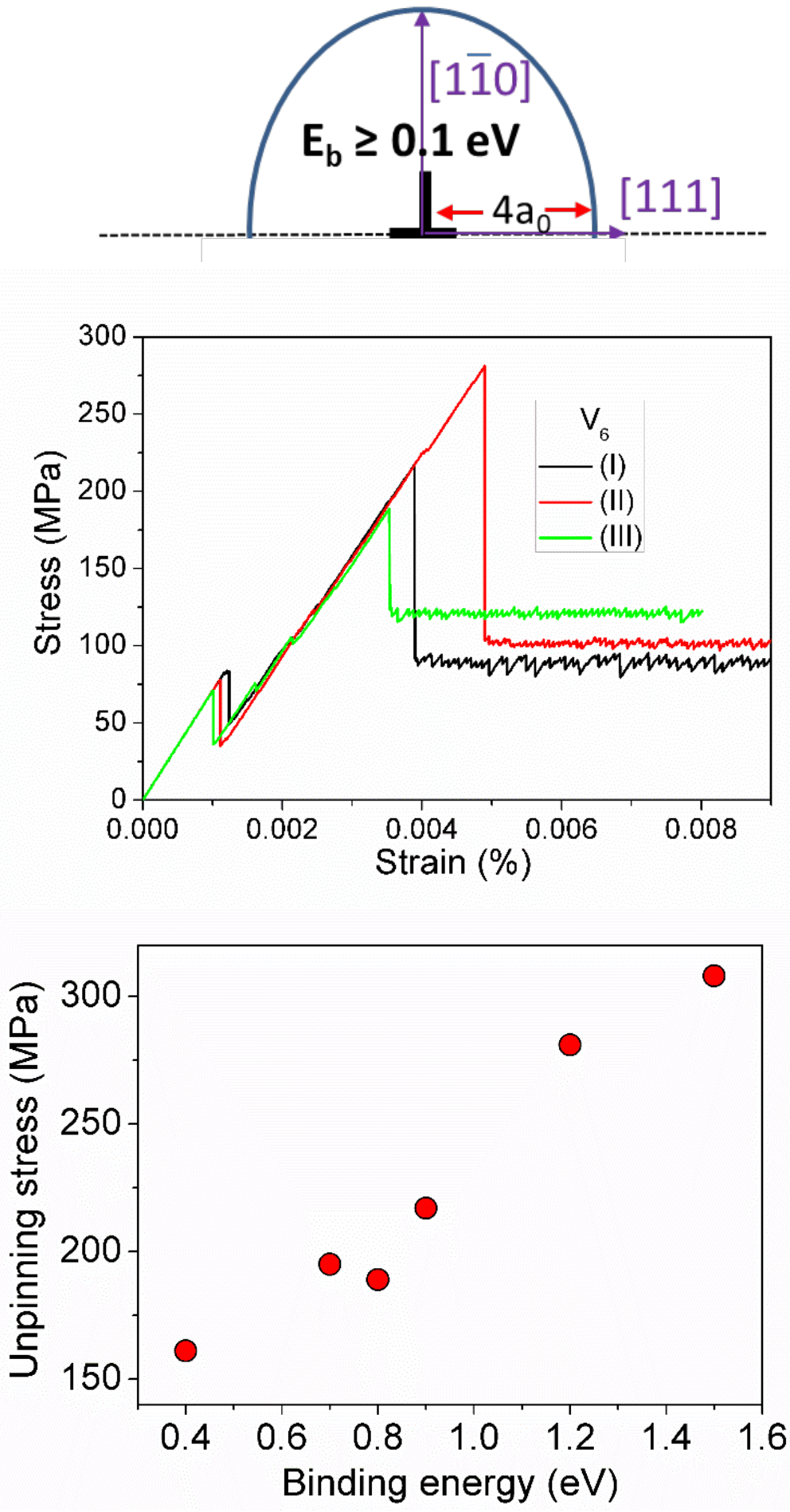

Figure 3. a) Schematics of the interaction region (binding energy $>0.1 \mathrm{eV}$ ) for vacancy clusters up to 6 vacancies. b) Stress-strain curves of a system formed by the edge dislocation and a $V_{6}$ cluster. The three curves correspond to the positions indicated in table 2. c) Unpinning stress of defects versus the defect-dislocation binding energy. 

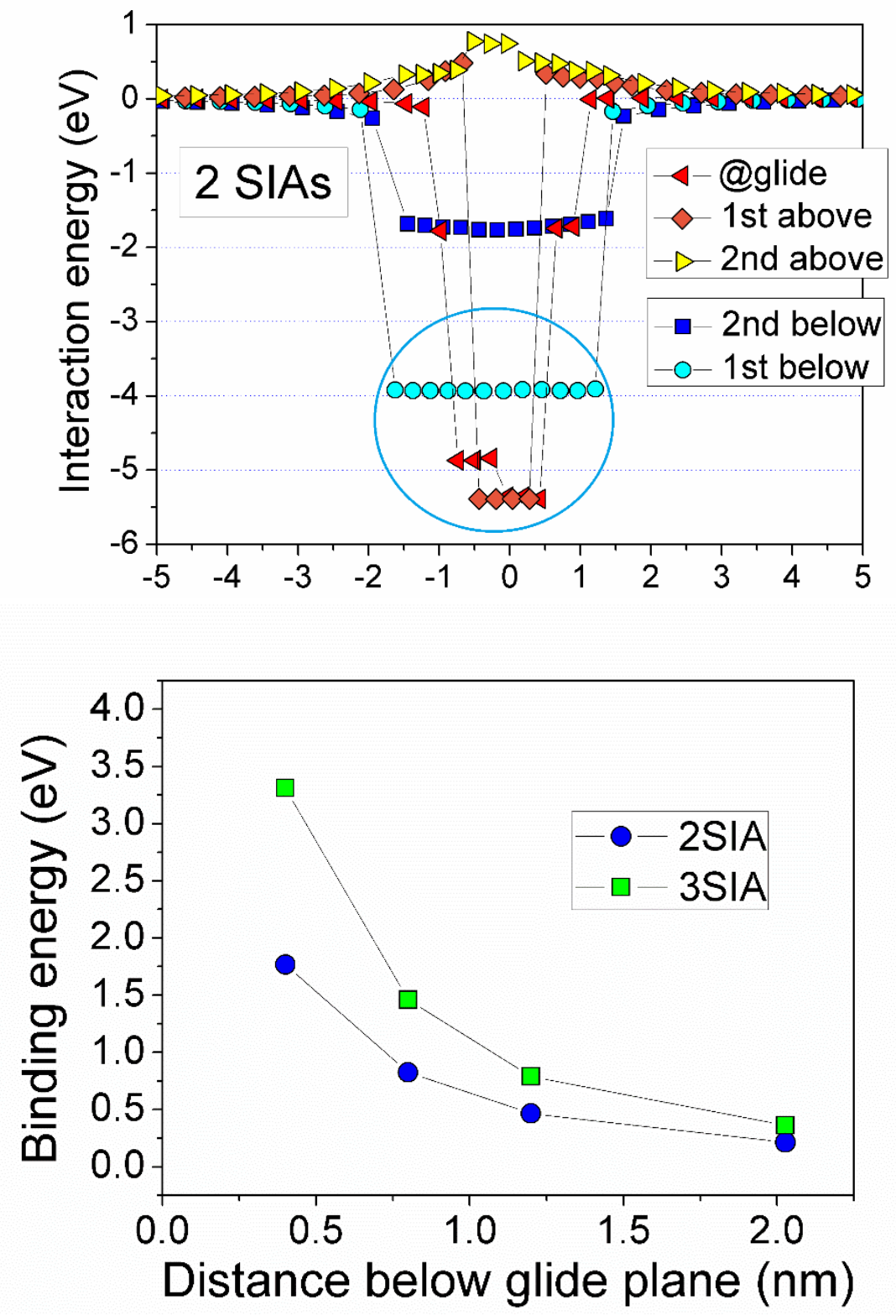

Figure 4. Interaction energy of 2SIA cluster with the edge dislocation versus the cluster position along the [111] direction. The colours of the curves refer to the position of the planes in figure 1a. Points encircled represent spontaneous absorption. Above and below refer to the dislocation glide plane, as in the caption of figure 2 . 

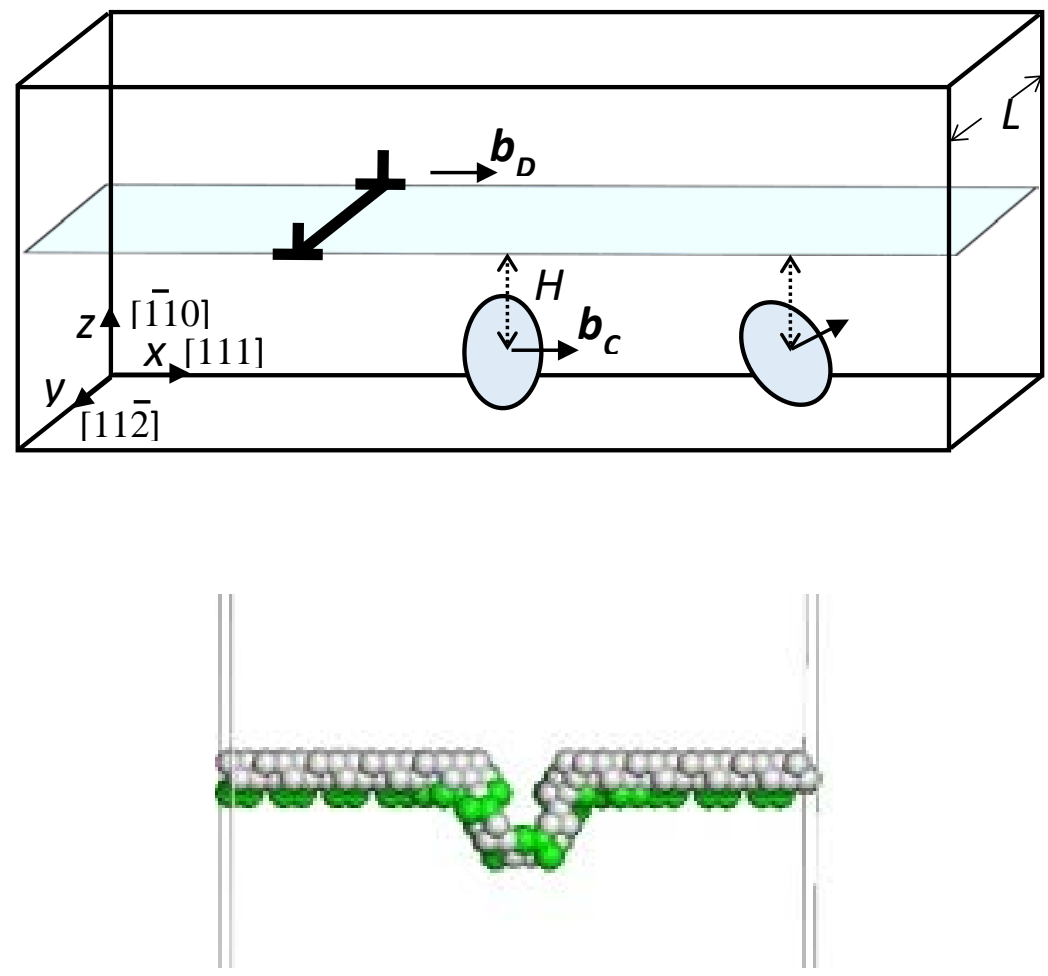

Figure 5. a) Schematics of the interaction of an edge dislocation with clusters of 7 and 19SIA. b) Dislocation line after the absorption of the 19SIA cluster. The atoms represented are chosen by their coordination number. 


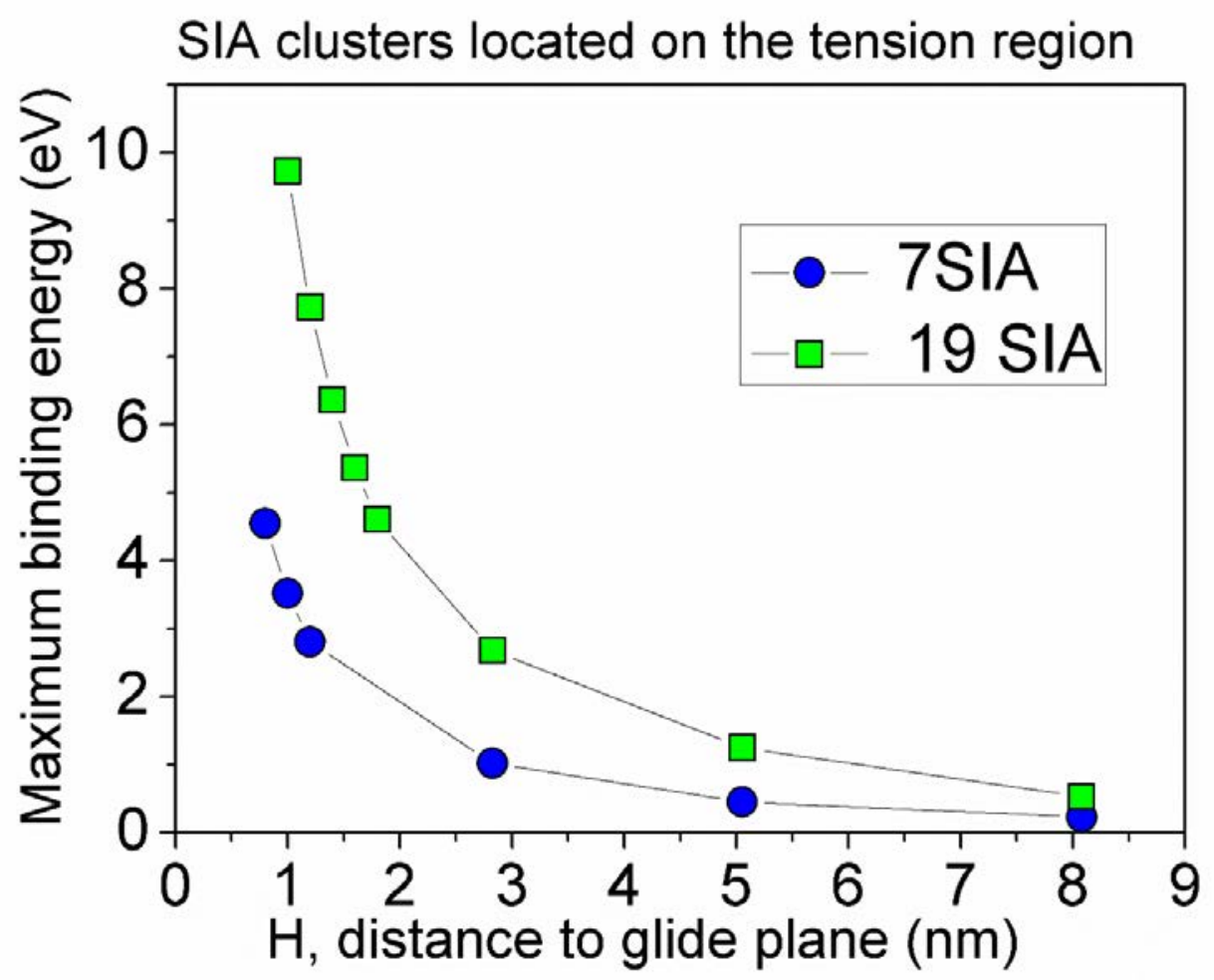

Figure 6. Binding energy of clusters of 7 and 19 SIA with Burgers vector parallel to the dislocation glide plane as a function of the distance from the centre of the cluster to the dislocation line. 